\title{
Effect of Increased Axial Field of View on the Performance of a Volume PET scanner
}

\author{
Joel S. Karp ${ }^{1}$, Paul E. Kinahan ${ }^{1}$, Gerd Muehllehner ${ }^{2}$, and Peter Countr/man ${ }^{2}$ \\ ${ }^{1}$ Department of Radiology, University of Pennsylvania, Philadelphia PA 19104 \\ 2UGM Medical Systems Inc., 3401 Market St., Philadelphia PA 19104
}

\section{DISCLAIMER}

\begin{abstract}
This report was prepared as an account of work sponsored by an agency of the United States Government. Neither the United States Government nor any agency thereof, nor any of their Government. Neither the United States Government, or assumes any legal liability or responsibility for the accuracy, completeness, or usefulness of any information, apparatus, product, or process disclosed, or represents that its use would not infringe privately owned rights. Reference herein to any specific commercial product, process, or service by trade name, trademark, manufacturer, or otherwise does not necessarily constitute or imply its endorsement, recommendation, or favoring by the United States Government or any agency thereof. The views and opinions of authors expressed herein do not necessarily state or reflect those of the United States Government or any agency thereof.
\end{abstract}

Presented at the IEEE Nuclear Science Symposium / Medical Imaging Confer ence November 2-9, 1991. Santa Fe, New Mexico

Address for correspondence:

Joel S. Karp, Ph.D.

Department of Radiology, Hospital of the University of Pennsylvania Nuclear Medicine, Donner 110 3400 Spruce St.

Philadelphia, PA 19104

- MASTER

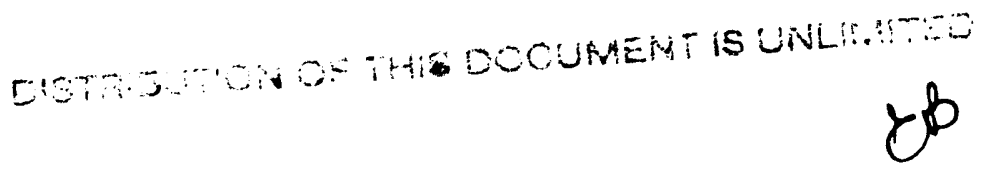

$$
\text { FGO2-88ERGOG42 }
$$




\title{
Effect of Increased Axial Field of View on the Performance of a Volume PET scanner
}

\author{
Joel S. Karp' 1 , Paul E. Kinahan 1 , Gerd Muehllehner ${ }^{2}$, and Peter Countryman ${ }^{2}$ \\ ${ }^{1}$ Department of Radiology, University of Pennsylvania, Philadelphia PA 19104 \\ 2UGM Medical Systems Inc., 3401 Market St., Philadelphia PA 19104
}

\begin{abstract}
The performance of the PENN-PET $240 \mathrm{H}$ scanner from UGM Medical Systems is tested and compared to the prototype PENN-PET scanner built at the University of Pennsylvania. The UGM PENN-PET scanner consists of six continuous position-sensitive $\mathrm{NaI}(\mathrm{Tl})$ detectors, which results in a 50-cm transverse fie'd-of-view and a $12.8-\mathrm{cm}$ axial fieldof-view. The fine spatial sampling in the axial direction allows the data to be sorted into as many as 64 transverse planes, each 2 -mm thick. A large axiai acceptance angle, without interplane septa, results in a high sensitivity, with a low scatter and randoms fraction. due to the use of a narrow photopeak energy window. This paper emphasizes those performance measurements that illustrate the special characteristics of a volume imaging scanner and how they change as the axial length is increased.
\end{abstract}

\section{INTRODUCTION}

Historically, positron emission tomography (PET) scanners have developed as two-dirnensional systems. beginning as a single ring of dicectors and evolving into multiple stacks of rings, separated by lead shielding. By mechanically eliminating photons that are not emitted parallel to the plane of detectors, PET was able to take advantage of the two-dimensional reconstruction algorithms developed for computed tomography (CT), where photon paths are constrained to lie in a prederermined plane. Mathematically, the in-plane events provide enough information to reconstruct the object in PET. However, the image quality suffers in many cases because of poor statistics, since only about $1 \%$ of the total events emitted isotropically from the source of activity are used. It is for this reason that volume imaging has received considerable attention lately.

The PENN-PET scanner [1] developed at the University of Pennsylvania and completed in 1987 was the first multi-slice PET system built without inter-ring septa, resulting in a large axial acceptance angle. During its development, this was a departure from most other PET systems that were based on discrete BGO detectors in a multi-ring configuration. Presently, most BGO systems have now incorporated some degree of position encoding, such as the block-detector design [2], and some BGO systems are now being built with either retractable septa [3] or without any septa at all [4]. The purpose of inter-ring septa is two-fold. The first reason, as stated above, is to constrain the detected photons to lie in a plane, thus allowing the use of a two-dimensional reconstruction algorithm. Therefore, without septa, we expected to see some degradation of image quality with a large axial acceptance angle, particularly at a large radial distance from the center of the scanner. We have since come to the conclusion that the degradation in imara quality is not significant for most studies, although a three-dimensional reconstruction algorithm does have advantages. The second reason for the septa is to limit the acceptance of scattered radiation from the patient. Sinie we use $\mathrm{NaI}(\mathrm{TI})$ detectors that have better than $10 \%$ energy resolution (FWHM), we can acquire data with a high energy threshold of $450 \mathrm{keV}$. This limits the scatter fraction (scatter/total) to $13 \%$ for brain imaging.

Based on the proto-type PENN-PET scanner, a second PENN-PET scanner was built by UGM Medical Systems and delivered to the University of Pennsylvan:a in $1 \neq 90$. These scanners are used for both brain (18 F-fluoro-deoxy-glucose, ${ }^{15} \mathrm{C}-\mathrm{H}_{2} \mathrm{O},{ }^{18} \mathrm{~F}$-n-methyl-spiperone) and cardiac ( ${ }^{18} \mathrm{~F}$-fluorodeoxy-glucose, ${ }^{82} \mathrm{Rb}$ ) studies. The UGM scanner was built with a larger axial field-of-view (FOV), $12.8 \mathrm{~cm}$ compared to $9.0 \mathrm{~cm}$ for the proto-type scanner, since we did not seem to be limited by the two-dimensional reconstruction or the scattered radiation. The larger FOV, however, results in twice the sensitivity and allows the entire brain or hear to be imaged, without any axial truncation. The PENN-PET system offers high spatial resolution in all three directions, high sampling density along all three axes without scanner motion, and good energy and timing resolution. The high spatial resolution and sampling density result in the three-dimensional imaging capability.

This paper compares the performance of the two scanners to point out the advantages of increasing the axial FOV. In addition, preliminary results of a three-dimensional reconstruction algorithm are shown to illustrate the limitations of the two-dimensional recorstruction. Finally, plans are discussed for a scanner with an axial FOV of $25 \mathrm{~cm}$, based on the PENN-PET design, which will result in much higher sensitivity.

\section{PENN-PET SCANNER}

\section{Dezectors and Electronics}

The PENN-PET scanner design uses six continuous 2dimensionally position-sensitive $\mathrm{NaI}(\mathrm{Tl})$ cetectors with a high 
degree of position encoding. Each detector is $50 \mathrm{~cm}$ long (transverseiy) and is coupled to $5-\mathrm{cm}$ square photomultiplier tubes (PMTs). The major difference between this detector and the one used in the proto-type scanner is the larger crystal size axially, $14 \mathrm{~cm}$ vs. $10 \mathrm{~cm}$ (see Figure 1), and the
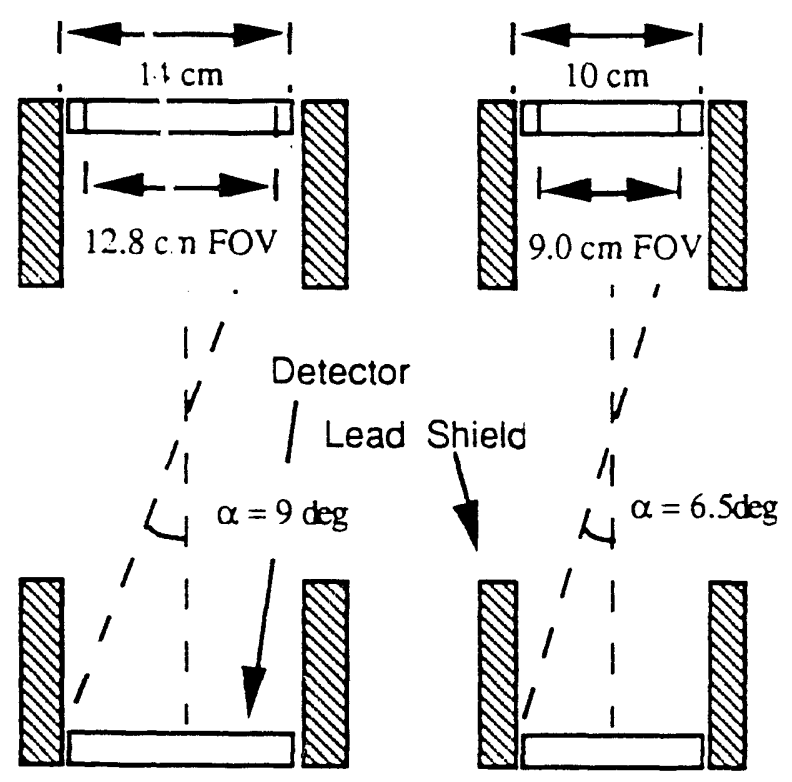

Figure 1. A) PENN-PET scanner. The axial FOV is 12.8 $\mathrm{cm}$ for the UGM scanner (four rows of PMTs) and $9.0 \mathrm{~cm}$ for the proto-type scanner (three rows of PMTs). The transverse FOV is $50 \mathrm{~cm}$ for both scanners.

larger number of PMTs per detector, 40 vs. 30 . Although the crystal size increases by about $50 \%$, the total number of PMTs increases by only $33 \%$. The proto-type design allowed only a 9-cm axial field-of-view (FOV), which was marginal in terms of imaging the entire brain or heart. The larger detector allows a 12.8-cm axial FOV, which makes a noticeable improvement in sensitivity as well. Rather than a maximum of 45 transverse slices (each 2-mm thick) for the original system, the new system can image a maximum of 64 slices. Both systems can resort the data into a maximum of 128 sagittal or coronal slices.

The PMT signals are pulse-shortened, digitized and integrated for $240 \mathrm{~ns}$, and then sent to a position calculator that determines the local centroid positions (transverse and axial) of the coincident events. The electronics and position algorithm have been described previously [1] in detail. One difference in the UGM scanner which leads to an improvement in performance is the use of 8-bit Analog to Digital Converters (ADC) instead of the original 6-bit ADC's. Another difference is the incorporation of local coincidence triggering [5], which electronically increases the number of detectors and coincident channels in the system. This was developed to improve the countrate capability of the system and to make it possible to build a cylindrical scanner that consists physically of only one detector. This will be further discussed in Section IV.

\section{Reconstruction Algorithm}

The data from the PENN-PET are normally rebinned (in hardware) into two-dimensional sinograms (256 rays $x 192$ angles), whose slice number is determined by averaging the axial coordinates of the two coincident detectors [6]. This single-slice rebinning method is a geometrical approximation for oblique rays, except for those events originating at the center of the scanner. The slices (up to 64 for the UGM scanner) are then reconstructed independently by filtered backprojection, with corrections for efficiency normalization, scattered and random coincidences, attenuation, and gap compensation [1]. In addition, an axial normalization is performe 1 to compensate for the non-uniform axial sensitivity.

A new three-dimensional algorithm is also used, multislice rebinning [7], that achieves a higher degree of geometrical accuracy for oblique rays without the computational burden of a fully three-dimensional algorithm. This approach rebins oblique rays into multiple sinograms, depending on how many are intersected by the coincident line. The data are then reconstructed two-dimensionally, with the same quantitative correction methods as those applied above. This step is immediately preceded or followed by axial filtering to reduce the blurring in the axial direction, which is independent of the filtering in the transverse direction during reconstruction.

Finally, a fully three-dimensional algorithm is used [8] that requires the data to be spatially invariant. This is accomplished by estimating missing data by reprojecting through a two-dimensional reconstruction of the object. The missing data are due to the finite axial extent of the scanner that occurs with every practical scanner, as well as the gaps between the detectors, which is a characteristic of the PENNPET. While this algorithm is more accurate for oblique rays, it requires more computation and different methods of quantitative corrections.

Neither 3-D algorithm is implemented in hardware yet, and so the data must be taken in list mode. While this is useful to test the algorithms with point source data and a limited number of phantoms, it is not practical to acquire list mode data for a patient study, because of the limitations on disk transfer time and disk storage space.

\section{SCANNER PERFORMANCE}

\section{Spatial Resolution}

For the measurement of the image spatial resolution a small point source in air is used, placed at several transverse and axial positions. With a 3-D imaging system, the transverse and axial resolution can be mitasured simultaneously with a point source.

The image resolution depends not only on the reconstruction algorithm and filter, but also the sampling density. With the PENN-PET, the axial sampling can be decreased to $2 \mathrm{~mm}$, but the transverse sampling can be decreased to $0.5 \mathrm{~mm}$. For a typical brain study we use 2 -mm 
sampling in both the transverse and axial directions, but for a cardiac study we use 4-mm sampling in both directions. For a small animal study, we use $1-\mathrm{mm}$ sampling in the transverse direction, and 2-mm sampling in the axial direction. For the measurement of intrinsic syatial resolution, we varied the sampling size, to investigate its effect. In the center of the FOV, with the axial sampling fixed at $2 \mathrm{~mm}$, the transverse spatial resolution is $4.9 \mathrm{~mm}, 5.6 \mathrm{~mm}$, and $6.2 \mathrm{~mm}$ for a transverse sampling size of $1 \mathrm{~mm}, 2 \mathrm{~mm}$, and $4 \mathrm{~mm}$, respectively. The axial resolution is $5.6 \mathrm{~mm}$, with $2-\mathrm{mm}$ sampling size.

With the two-dimensional single-slice rebinning (2D. SSRB) method, we expect a loss of axial resolution as the distance from the center of the scanner increases. The tangential resolution (perpendicular to the radial direction in the transverse plane) also suffers, because the projection data become inconsistent as events are misplaced axially into other slices preferentially at angles parallel to the radial direction of the source. At the center of the FOV of the scanner the axial resolution (FWHM) is $5.6 \mathrm{~mm}$, but increases to $13 \mathrm{~mm}$ at a radius of $10 \mathrm{~cm}$ with the full acceptance angle of the UGM scanner of $+1-9.0 \mathrm{deg}(\alpha=9 \mathrm{deg})$, or $10 \mathrm{~mm}$ with the full acceptance angle of the proto-type scanner of $+/-6.5 \mathrm{deg}(\alpha=$ $6.5 \mathrm{deg}$ ), as seen in Figure 2. Near the edge of the axial FOV, however, where the axial acceptance angle is geometrically restricted, the spatial resolution does not vary significantly. Although the degradation in resolution in the center can be minimized by limiting the axial acceptance angle over the whole FOV (in software) this would lead to a reduction in the system sensitivity.

A 3-D reconstruction algorithm, however, promises to preserve both the spatial resolution and system sensitivity of a volume imaging scanner with a large axial acceptance angle. The point source data were therefore reconstructed with both the multi-slice rebinning (3D-MSRB) algorithm and the reprojection (3D-RP) algorithm. The 3D-MSRB algorithm leads to a more uniform axial resolution as a function of radius compared to the 2D-SSRB, being slightly worse in the center, but considerably better at $10 \mathrm{~cm}$. The 3D-RP algoritt.m leads to even more uniform resolution, with the axial resolution comparable to the transverse resolution for all radii.

\section{Scatter Fraction}

In addition to defining the planes in a multi-ring scanner, the septa also serve to reduce the arnount of scattered radiation which is detected. The PENN-PET scanner has no septa.

However it has been shown that with good energy resolution, a high energy window is as effective as septa at reducing scatter. An energy resolution (FWHM) of $10 \%$ is achieved with the $\mathrm{NaI}(\mathrm{Tl})$ detectors, with the same pulseshortening and $240 \mathrm{~ns}$ integration time used to obtain high countrate capability.
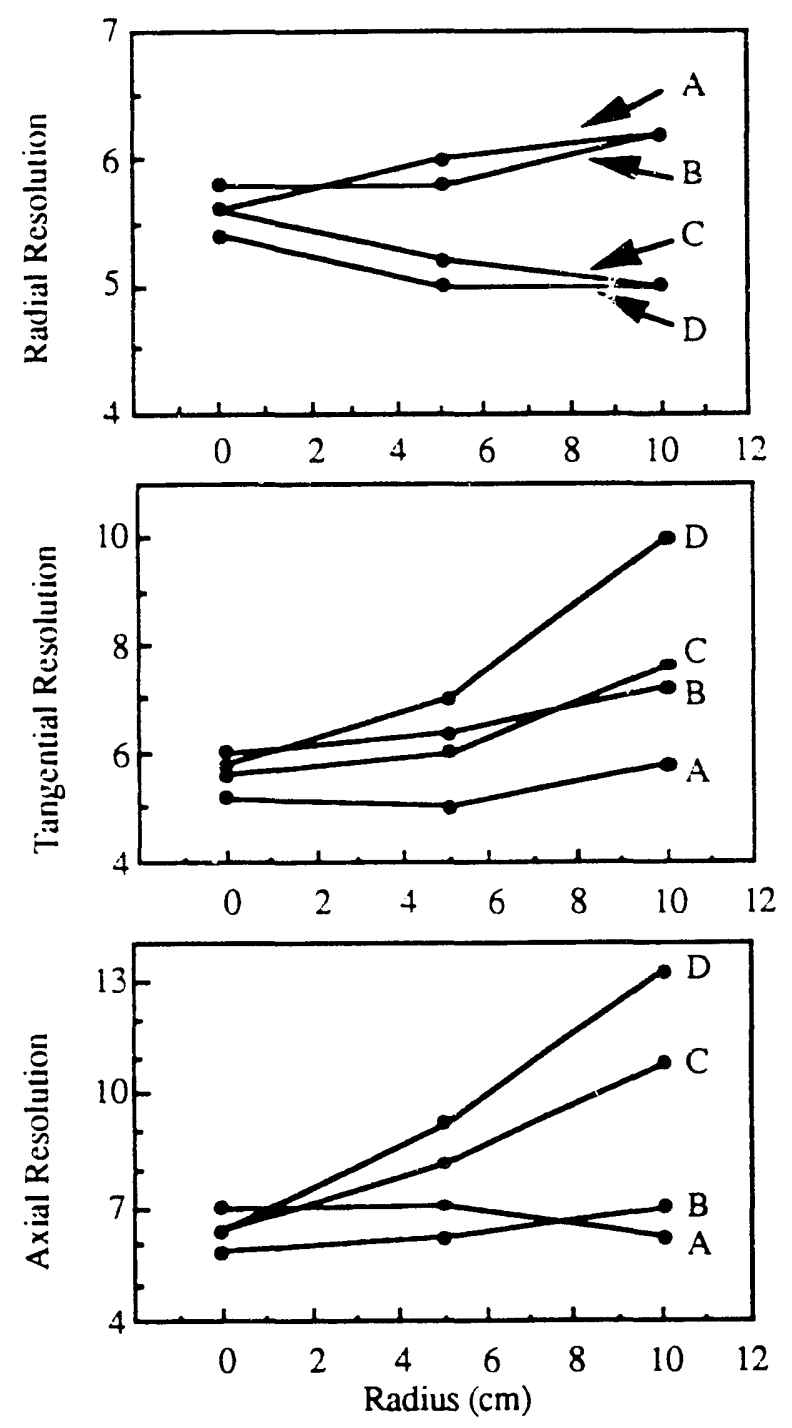

Figure 2. Radial, tangential, and axial spatial resolution (FWHM in $\mathrm{mm}$ ) as a function of radius (cm) for 2D and $3 \mathrm{D}$ reconstruction algorithms as explained in the text. Note the change in scale. These data are reconstructed with $2-\mathrm{mm}$ transverse and axial sampling. The algorithms are designated: A: MSRB, B: 3D-RP, C: SSRB (6.5 deg), D: SSRB (9 deg).

With a uniform distribution of activity in a head-sized phantom, $18-\mathrm{cm}$ diameter by $10-\mathrm{cm}$ long, the scatter fraction (scatter/total) is measured as a function of axial aperture with the lower-level energy threshold set at $450 \mathrm{keV}$. The scatter fraction is seen to increase from $7 \%$ with an axial aperture of 2-cm to $13 \%$ for the proto-type PENN-PET (axial aperture of $10-\mathrm{cm}$ ) and UGM PENN-PET (axial aperture of $14-\mathrm{cm}$ ), as seen in Figure 3. Although these values represent an average 


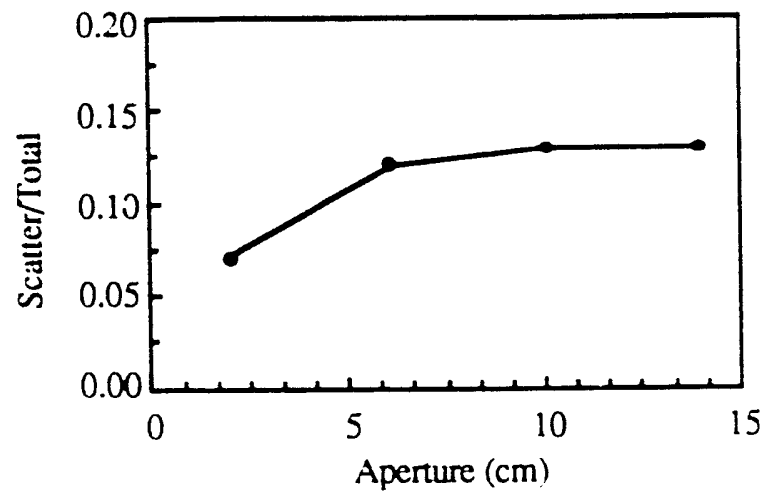

Figure 3. Scatter fraction vs. axial aperture

over the axial FOV, we find that the scauter fraction does not change significantly as a function of slice location despite the large acceptance angle and lack of septa. In other words, the axial sensitivity profile of the scattered radiation is similar to that of the true events. While the slices near the edge of the axial FOV suffer from poorer sensitivity than those near the center, because of the smaller axial acceptance angle, the relative amount of scatter to true events does not increase.

If the phantom size is increased to that proposed to be a standard [9] for performance measurements (20-cm diameter by $18.5-\mathrm{cm}$ long), the scatter fraction increases to about $20 \%$. This is due to the additional activity outside the FOV which is not completely ,nielded from the detectors. For a brain study, however, there is no activity above the brain and the activity below the brain (in the heart and lungs) is far enough outside the FOV to be effectively shielded. Even for cardiac studies that usually have some activity outside the FOV in the lungs and liver, we find that the scatter fraction is less than that measured with the $20-\mathrm{cm}$ phantom. The $20-\mathrm{cm}$ phantom, which is meant to be a compromise between the head and the body, therefore does not necessarily predict the same amount of scattered radiation that is encountered in a patient study. Although a standard size is needed for comparison measurements among different scanners, which was the goal of the Society of Nuclear Medicine and NEMA groups [9], the results still are affected by the axial FOV of the scanner and the shielding design.

\section{Sensitivity}

The system true sensitivity of the UGM scanner, as measured with the $20-\mathrm{cm}$ phantom, is $130 \mathrm{kCPS} / \mu \mathrm{Ci} / \mathrm{cc}$, with an energy threshold of $450 \mathrm{keV}$ and a maximum axial acceptance angle of $\alpha=6.5 \mathrm{deg}$. This value reflects the result of subtracting the estimated scatter from the measured total. The system sensitivity is $65 \mathrm{kCPS} / \mu \mathrm{Ci} / \mathrm{cc}$. Although $\mathrm{NaI}(\mathrm{Tl})$ has intrinsically lower stopping power than BGO, the large axial acceptance angle of the PENN-PET system results in an overall sensitivity comparable to a multi-ring BGO system, with higher slice sensitivity near the center, and lower near the ends.

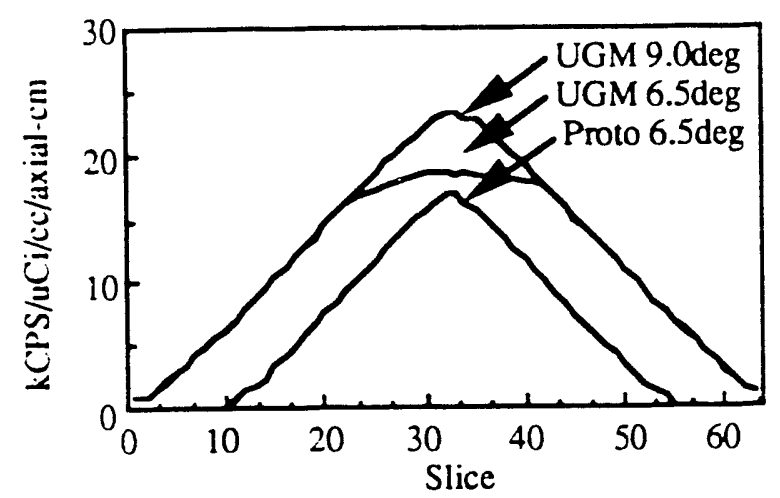

Figure 4. Axial sensitivity profiles (vs axial acceptance angle)

Figure 4 shows the axial sensitivity profiles, for the UGM scanner with the full axial acceptance angle of $\alpha=9 \mathrm{deg}$, and for the proto-type scanner, with $\alpha=6.5 \mathrm{deg}$. The profile is also shown for the UGM scanner with a software limit of $\alpha=$ $6.5 \mathrm{deg}$, which is used to reduce the axial blurring at large radii when employing the $2-D$ reconstruction algorithm. For patient studies, the data are normally smoothed axially by addi:-:othree $2-\mathrm{mm}$ slices together, but retaining a 2-mm axial sampling. The measured slice sensitivity, for a $6-\mathrm{mm}$ slice thickness, peaks in the center at $14 \mathrm{kCPS} / \mu \mathrm{Ci} / \mathrm{cc}$ for $\alpha=9 \mathrm{deg}$ and 10 $\mathrm{kCPS} / \mu \mathrm{Ci} / \mathrm{cc}$ for $\alpha=6.5 \mathrm{deg}$. For the UGM scanner, only $12.8 \mathrm{~cm}$ of $14 \mathrm{~cm}$ axial FOV is used, and for the proto-type scanner $9.0 \mathrm{~cm}$ of $10 \mathrm{~cm}$ is used, so that the edge slices do not have a 'zero' sensitivity.

\section{Countrate Losses and Random Coincidences}

The countrate measurement was performed with the headsized phantom $(18-\mathrm{cm}$ by $10-\mathrm{cm})$, which gives an indication of the performance that is encountered for the brain studies for which the scanners are being used. Actually, this phantom (2.5 liters) is still considerably larger than most brains (about 1.3 liters), but it is more realistic than the $20-\mathrm{cm}$ phantom $(6.0$ liters). The countrate data are shown in Figure 5, along with the noise equivalent countrate, where NEC $=\mathrm{T} /(1+\mathrm{S} / \mathrm{T}+$ $\mathrm{R} / \mathrm{T}$ ). A comparison between the two scanners indicates that the true countrate saturates at a slightly higher countrate for the UGM scanner ( $48 \mathrm{kCPS}$ vs. $43 \mathrm{kCPS}$ ) although the UGM scanner, which is twice as sensitive, does so at a lower activity concentration $(1.0 \mu \mathrm{Ci} / \mathrm{cc}$ vs. $1.5 \mu \mathrm{Ci} / \mathrm{cc})$. It is also significant that the random fraction is much smaller for the larger scanner compared to the proto-type scanner. The random fraction as a function of true countrate is plotted in Figure 6 . The coincidence resolving time is $2 \tau=10 \mathrm{~ns}$ for both scanners.

The countrate limitation (where the true countrate curve turns over) for both scanners, is due mainly to two effects. The first major deadtime factor is electronic deadtime, due to the processing speed of the position calculator and on-line sinogram rebinning electronics. A large number of events are 

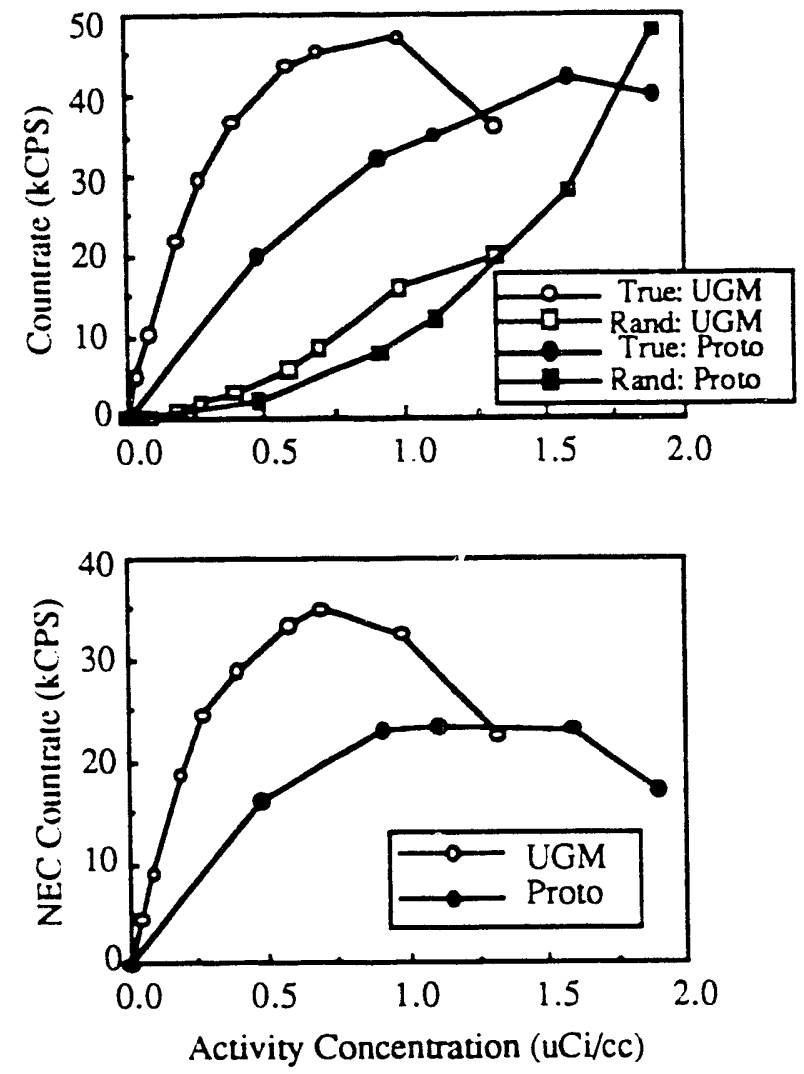

Figure 5. A) The true and random system countrates for the UGM scanner and the proto-type scanner, using the brainsized phantom, which is $18 \mathrm{~cm} \times 10 \mathrm{~cm}$. These are the rates within a $24-\mathrm{cm}$ transverse FOV, with scatter subtracted. B) The noise equivalent countrates for the two scanners.

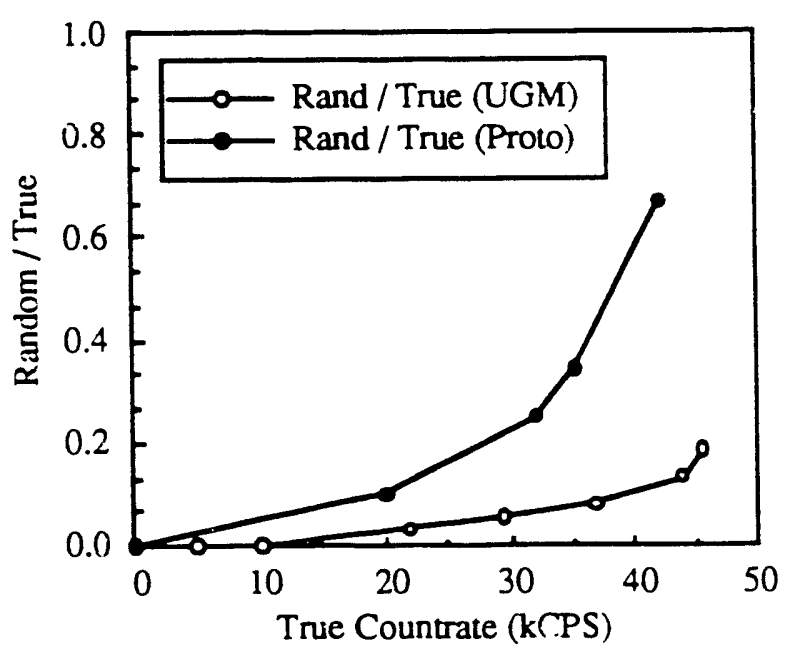

Figure 7. Random fraction vs. true countrate

processed and eventually eliminated due to requirements of the energy window, position distortion correction, and restricted field-of-view, so that at the saturation point at which the true countrate is $45 \mathrm{kCPS}$, the electronics are processing more than $200 \mathrm{kCPS}$. The local coincidence triggering system
[5] was designed to increase the number of effective detector channels, thus reducing the deadtime of each channel. The second major deadtime factor is caused by pulse pile-up in the detector. This results in energy pile-up and these events are eliminated by the energy window requirement. Pile-up can be further reduced by shortening the pulse integration time [10], however, this would also affect the intrinsic spatial resolution. Although $240 \mathrm{~ns}$ integration time is a good compromise for a wide dynamic range of countrates, the highest countrate studies may benefit from a shorter integration time.

\section{Image Quality}

While we are working on a practical 3-D reconstruction algorithm [7,8], we presently image patient data with the 2-D reconstruction and believe that the geometrical approximations of single-slice rebinning are not that significant for most imaging situations, at least with the axial extent of the present PENN-PET scanners. In order to evaluate the effect of the axial blurring for a distributed object, we imaged a phantom of alternating disks, with and without activity, each $1.25-\mathrm{cm}$ thick and $20-\mathrm{cm}$ in diameter, as seen in Figure 7. Figure 8A, $B, C$ plots the counts per slice for three images acquired with $\alpha=9 \mathrm{deg}, 6.5 \mathrm{deg}$, and $1 \mathrm{deg}$. The contrast (peak/valley) in the center, indicating the degree of axial mis-positioning, is 2.1 , $2.8,4.2$, for $\alpha=9 \mathrm{deg}, 6.5 \mathrm{deg}$, and $1 \mathrm{deg}$, respectively. The image with the small axial acceptance angle serves as the standard, since the axial mis-positioning is minimized. All images were acquired for the same total number of counts, and are not normalized for the non-uniform axial efficiency. The disk phantom was also reconstructed with the 3-D multi-slice rebinning algorithm with the acceptance angle unrestricted. As expected, the contrast, 3.7, is better than that of the 2-D algorithm, as shown in Figure 8D.

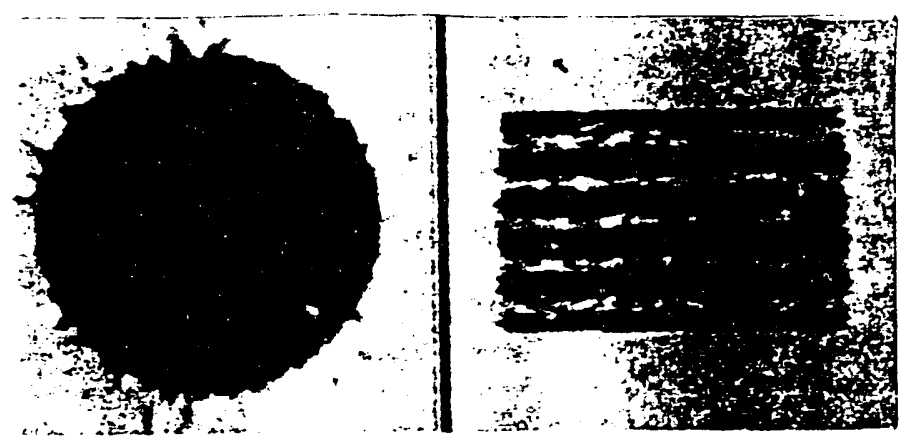

Figure 7. Transverse and coronal sections of disk phantom

In a previous experiment [11], we performed a signal-tonoise analysis (contrast/noise) using cold spheres in a uniform medium of activity, and varied both the energy threshold and axial acceptance angle, in order to evaluate the tradeoffs of sensitivity, scatter, and spatial resolution. The conclusions 

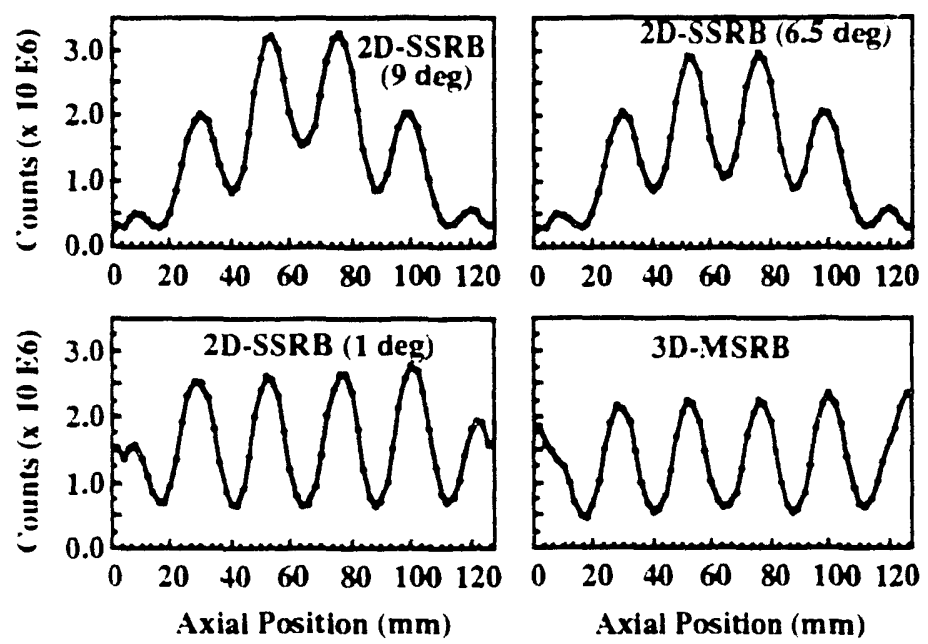

Figure 8. Axial profiles of disk phantom, reconstructed with A) 2D-SSRB (9deg), B) 2D-SSRB (6.5deg), C) 2DSSRB (1deg), and D) MSRB algorithms.

were that a high energy threshold ( $450 \mathrm{keV})$ leads to the best $S N R$, and that with the $2-D$ reconstruction algorithm, the images taken with a laige axial acceptance angle had a higher SNR than those taken with a small axial acceptance angle, for an equal imaging time. For equal counts, however, the images with a small axial acceptance angle had a higher SNR, but the difference was not striking. This second comparison implies that a 3-D algorithm, which preserves both the sensitivity and the spatial resolution, would lead to a moderate improvement in image quality, compared to the 2-D algorithm.

The degree to which a 3-D algorithm is necessary for a realistic brain study is illustrated in Figure 9. We used the 3-D brain phantom (Data Spectrum), which is composed of 19 different sections, each approximately $6-\mathrm{mm}$ thick, with a gray-to-white matter ratio of 4-to-1 within each section. The phantom was filled with ${ }^{18} \mathrm{~F}$ and the data were acquired with an acceptance angle $\alpha=6.5 \mathrm{deg}$ and $\alpha=1.5 \mathrm{deg}$, to minimize the geometrical approximation of $2 \mathrm{D}$ single-slice rebinning, and both data sets were reconstructed with the 2D-SSRB algorithm. The first image, with $\alpha=6.5 \mathrm{deg}$, was acquired for a time typical for ${ }^{18}$ F-FDG studies, 30 minutes, which results in a total of about 40 million counts. The second image, with $\alpha=1.5 \mathrm{deg}$ was acquired for 90 minutes, in order to collect the same number of counts with lower sensitivity. Visually, it is difficult to see the difference between these images, despite the poorer axial resolution with $\alpha=6.5 \mathrm{deg}$ particularly at the cortex, which is at a radius of approximately $10 \mathrm{~cm}$. These images are 6-mm thick axially (the sum of three $2-\mathrm{mm}$ slices), and include filtering in the transverse dirertion during filtered backprojection, which is how we normally process patient data. Although the phantom is ohviously not a real brain, it gives an indication that in a realistic imaging situation the improvement that a 3D algorithm has to offer over a 2D algorithm will not be dramatic. This is consistent with the previous experiment evaluating the SNR of cold spheres.

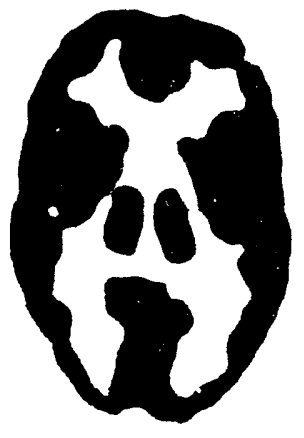

(A)

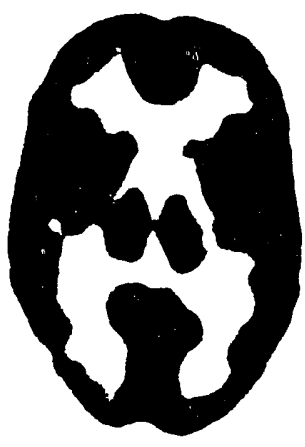

(B)

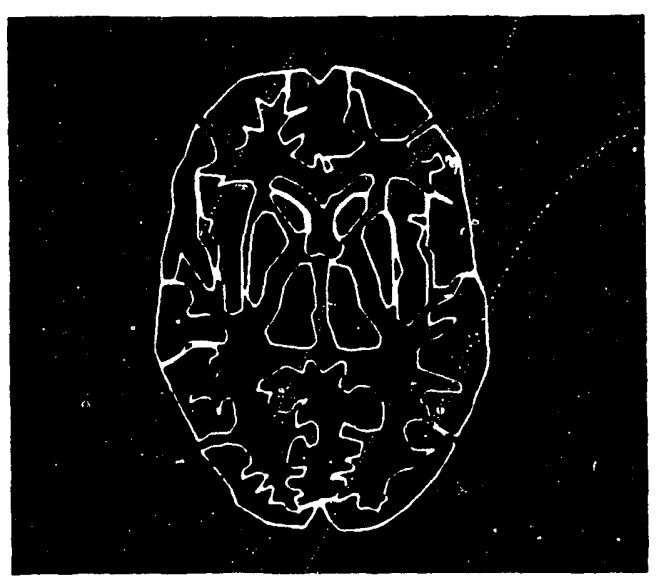

(C)

Figure 9. 3-D brain phantom images. A) Image with $\alpha=1.5 \mathrm{deg}$. B) Image with $\alpha=6.5 \mathrm{deg}$. C) Regions used for quantitative analysis.

A more quantitative comparison was made by fitting regions-of-interest (ROIs) over the suructures in the grey matter of this slice. A comparison between the counts in the ROIs of the two images shows a average difference of $1 \%$ and a maximum difference of only $5 \%$. These errors would be negligible compared to other errors present in a real patient study. For a larger axial acceptance angle than $\alpha=6.5 \mathrm{deg}$, however, it is likely that the need for 3D reconstruction will increase.

\section{Patient Studies}

Over the past year we have been performing a variety of studies on the UGM scanner, as well as on the proto-type scanner. A number of patients having ${ }^{18}$ F-FDG brain studies, were imaged sequentially on both scanners, in order to confirm that the two scanners give comparable quantitative results. The extended axial FOV of the UGM scanner is clearly advantageous (Figure 10) particularly since the PET images are often matched with an MRI image of the same patient. This 
patient received $8 \mathrm{mCi}$ and was imaged for 30 minutes in each scanner, beginning 40 minutes post-injection. The UGM
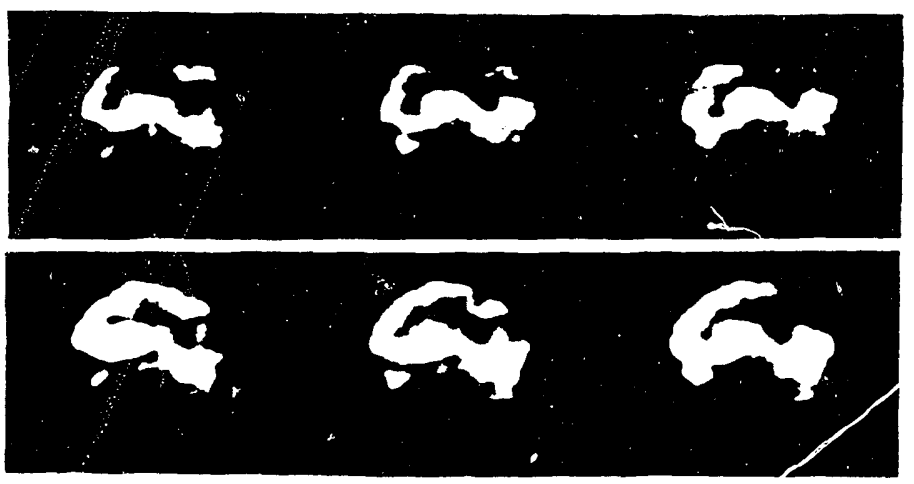

Figure 10. Sagittal brain sections of patient imaged with ${ }^{18}$ F-FDG with proto-type (top) and UGM scanners (bottom).

scanner, with its better high countrate capability, is also regularly used for bolus ${ }^{15} \mathrm{O}-\mathrm{H}_{2} \mathrm{O}$ stimulation studies. Rather than injecting a very shor bolus of high activity, which leads to countrate limitations of the scanner, a slow bolus technique [12] is being used, where $40 \mathrm{mCi}$ is injected over 3 minutes, and the data are collected dynamically in 10-20 second frames. Most of the data are then collected near the peak of the countrate curve shown in Figure 5.

The UGM scanner is also being used for cardiac studies, since this scanner has a fully implemented transmission scanning mode for attenuation correction. It uses a rotating rod source in which position is correlated with the coincident de:ection of events. Again, the advantage of the larger axial FOV is that patient positioning is not as critical and the sensitivity at the edge of the heart is better. We have imaged patients with ${ }^{18} \mathrm{~F}$-FDG, as well as ${ }^{82} \mathrm{Rb}$.

\section{CYLINDRICAL PET SCANNER}

In comparing the UGM scanner to the proto-type scanner, there were several expected results, as well as some that surprised us. It is clear that a larger axial FOV is advantageous in terms of imaging the entire brain or heart, particularly in practice where the technologist can not always center the organ precisely. The improved sensitivity follows naturally from the larger geometrical solid angle (Figure 4), but so does increased scatter and randoms. However, the scatter fraction does not increase, for a head-sized object, with good energy discrimination (Figure 3), and the random fraction decreases as a function of the true countrate (Figure 6). Finally, a 3-D reconstruction algorithm was shown to reduce the degradation in spatial resolution that is present with a 2-D algorithm, when data are collected without septa.

We therefore believe that we can extend this design even further in the axial dimension. An advantage of the PENNPET system, with continuous detectors, is that the increase in performance as a function of axial FOV is greater than the corresponding increase in cost and complexity. If we restrict our design to brain imaging, an axial FOV of $12.8 \mathrm{~cm}$ is large enough, and so an increase in the axial size of the detector will serve primarily to increase the sensitivity. By focusing on the brain, we do not have to be overly concerned about the scatter or random fractions increasing, since activity in the body will still be outside the FOV. With an increase of the size in the axial dimension to $25 \mathrm{~cm}$, and a decrease in the diameter to 42 $\mathrm{cm}$, the sensitivity will improve by a factor of ten over the proto-type scanner. In fact, the sensitivity at the edge of the brain would be higher than that of the UGM scanner at the center of the brain, as seen in Figure 11.

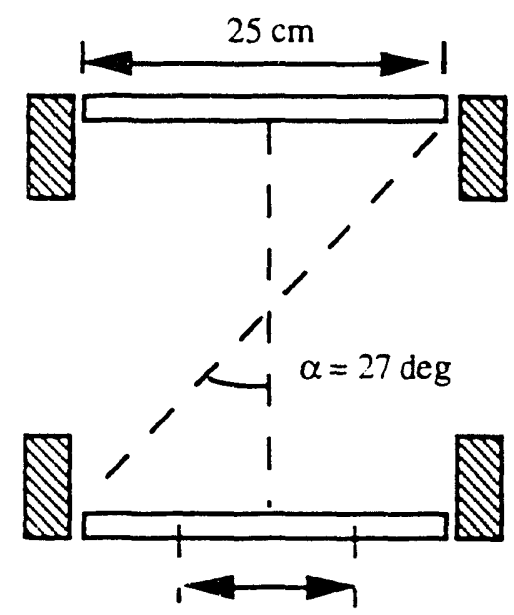

$12.8 \mathrm{~cm}$ FOV

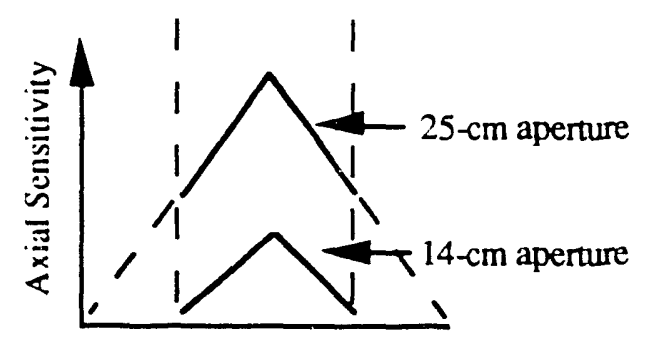

Figure 11. Schematic of large axial FOV PET scanne:

We are planning to build this scanner as a cylindrical system, using a single detector. The edge effects of the PENNPET detectors has always been an important consideration. although the Constrained Fourier Reconstruction (CFR) gap compensation technique [1] has allowed us to keep the system stationary during data acquisition. After considering the technical and practical constraints of eliminating the edge effects of position-sensitive detectors [13], we decided that we would achieve the best results with a cylindrical detector, which has no edge effects at all. In addition, with the local coincidence triggering system [5], it is now possible to electronically divide a continuous detector into separate channels, thus identifying coincidences in a single crystal.

The detector will be $1.9-\mathrm{cm}$ thick and coupled to thirty columns and six rows of 5-cm square PMTs, for a total of 180 PMTs. The thinner crystal, compared to the $2.5-\mathrm{cm}$ thick PENN-PET detectors, is expected to be about $17 \%$ less efficient, leading to $33 \%$ less coincident detection efficiency. 
However, the tremendous gain in geometrical efficiency will make up for this loss. In addition,we expect a significant improvement in the spatial resolution, since the thinner crystal will have less spatial resolution degradation due to Compton scattering and the spread of the light distribution.

\section{SUMMARY AND DISCUSSION}

The comparison of the UGM PENN-PET with the prototype PENN-PET illustrates the consequences of increasing the axial field-of-view with a scanner that does not use septa. An increase in the axial length of the detector from $10 \mathrm{~cm}$ to 14 $\mathrm{cm}$ leads to an increase in system sensitivity of a factor of two, from 65 to $130 \mathrm{kCPS} / \mu \mathrm{Ci} / \mathrm{cc}$, with 16 $\mathrm{kCPS} / \mu \mathrm{Ci} / \mathrm{cc} / \mathrm{axial}-\mathrm{cm}$ in the center. This is achieved with a maximum axial acceptance angle of $\alpha=6.5 \mathrm{deg}$, which is the limit of the proto-type scanner in the center. The main reason for limiting the acceptance angle with the UGM scanner is to limit the degradation in spatial resolution at large radii when using a two-dimensional reconstruction algorithm. With a three-dimensional algorithm, the acceptance angle can be increased to $\alpha=9 \mathrm{deg}$ in order to increase the sensitivity near the center to $24 \mathrm{kCPS} / \mu \mathrm{Ci} / \mathrm{cc} / \mathrm{axial}-\mathrm{cm}$. In addition, the spatial resolution is much more uniform, 5 to $7 \mathrm{~mm}$, out to a radius of $10 \mathrm{~cm}$. A method of efficiently implementing a 3-D reconstruction algorithm is being investigated.

With the larger axial FOV, the scatter does not increase for a head sized phantom. For an energy threshold of $450 \mathrm{keV}$, the scatter fraction is $13 \%$. In addition, neither the scatter fraction nor random fraction change significantly with slice location, since both the scatter and randoms have an axial profile similar to the true axial profile. The random fraction was shown to decrease significantly, as a function of true countrate, for the larger UGM scanner. Therefore, for a given activity, the larger UGM scanner will produce a higher true countrate than the proto-type scanner, because of the higher sensitivity, with a lower randoms fraction. Alternatively, a lower activity level with the UGM scanner can be used to produce the same true countrate, also with a lower randoms fraction. The high countrate limitations, caused by electronic deadtime, are currently being investigated.

In addition, since we have shown the larger scanner to offer significant advantages, we have considered extending the axial extent of the scanner even further, to $25 \mathrm{~cm}$, while restricting the imaging to the brain (or animals). This will lead to a large increase in sensitivity, better spatial resolution, a lower random fraction, and a comparable scatuer fraction and countrate capability.

\section{ACKOWLEDGEMENTS}

We would like to thank Robert Lewitt for his advice and the use of his Multi-Slice Rebinning algorithm. This work was supported by DOE grant number DE-FG()2-88ER60642.

\section{REFERENCES}

[1] Karp JS, Muehllehnet G. Mankoff DA, et al. Continuousslice PENN-PET: A positron tomograph with volume imaging capability. J Nucl Med 31: 617-627, 1990.

[2] Nutt R. Casey M. Caroll LR. A new multi-crystal two dimensional detector block for PET. J Nucl Med p28. 1985.

[3] Bailey DL, Jones T, Spinks TJ, Gilardi M-C, and Townsend DW. Noise equivalent count measurements in a neuro-PEI scanner wish retractable septa. IEEE Trans Med Imaging 10: 236-260, 1991

[4] Burnham CA. Kaufman DE. Chesler DA et al. Cylindrical PET detector design. IEEE Nucl Sci NS-35: 676-680, 1988.

[5] Mankoff DA. Muehllehner G, Miles GE. A local coincidence triggering system for PET tomographs composed of large. area position-sensitive detectors. IEEE Trans Nucl Sci NS. 37: $730-736,1990$.

[6] Daube-Witherspon ME, Muehllehner G. Trearment of axial data in three-dimensionai PET. J Nucl Med 28: 1717-1724. 1987.

[7] Lewitt RM, Muehllehner G, Karp JS. 3D image reconstruction for $P E T$ by multi-slice rebinning and axial filtering. (submitted to this conference)

[8] Kinahan PE. Karp JS. Three dimensional image reconstruction by reprojection. International conference of the IEEE engineering in medicine and biology society 12 : 383-384, 1990.

[9] Karp JS, Daube-Witherspoon ME, Hoffman EJ, et al. Performance standards in positron emission tomography. J Nucl Med. (in press)

[10] Mankoff DA, Muehllehner G, Karp JS. High countrate performance of a two-dimensionally position-sensitive detector for positron emission tomography. Phys Med Biol 34: 437-456, 1989.

[11] Karp JS, Kinahan PE, Mankoff DA. Positron emission tomography with a large axial acceptance aigle: Signal-tonoise considerations. IEEE Trans Med Imaging 10: 249-255. 1991

[12] Lammertsma AA, Cunningham MP, Heather JD et al. Combination of dynamic and integral methods for generating reproducible Ci3F images. J Cereb Blood Flow Metab 10: 675-686, 1990

[13] Freifelder RH, Haigh AT, Karp JS. Reducing edge effects and improving position resolution in position sensitive $\mathrm{Nal}(\mathrm{Tl})$ detectors. (submitted to this conference) 

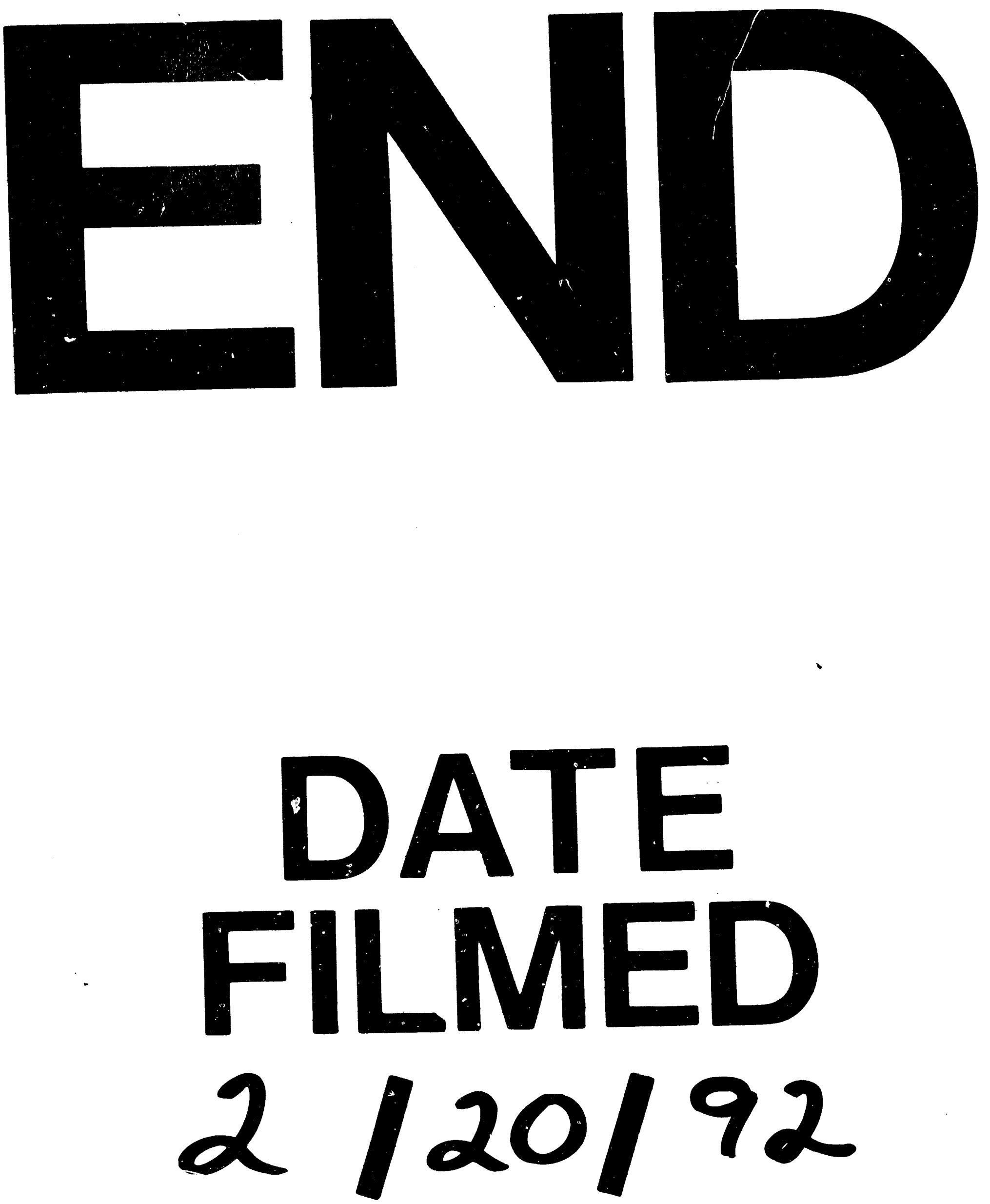
